\title{
LICHEN AND LICHENICOLOUS FUNGUS RECORDS FROM SANTA MARIA, AZORES ARCHIPELAGO
}

\author{
Pieter P.G. VAn den Boom
}

Arafura 16, 5691 JA, Son, the Netherlands

E-mail: pvdboom@kpnmail.nl

\begin{abstract}
Van den Boom P.P.G., 2021: Lichen and lichenicolous fungus records from Santa Maria, Azores archipelago. Botanica, 27(1): 34-43.

One hundred seventy-seven species of lichens and lichenicolous fungi are recorded for the island Santa Maria (Azores Archipelago, Portugal). Eighteen species are new for the archipelago. A list of all pertinent specimens with their locality and substrate is presented. Six rare species are illustrated.
\end{abstract}

Keywords: biodiversity, ecology, Macaronesia, lichens of Portugal, new records, taxonomy.

\section{INTRODUCTION}

The volcanic Azores archipelago, situated in the northern Atlantic Ocean, comprises 9 inhabited islands. The main island, São Miguel, is situated $1360 \mathrm{~km}$ west of continental Portugal. The climate is mild with average daily temperatures of $16^{\circ}-25^{\circ} \mathrm{C}$, and the temperature is never below $4{ }^{\circ} \mathrm{C}$. The average annual rainfall increases from east to west from below $800 \mathrm{~mm}$ on the southeasternmost island Santa Maria, to $6300 \mathrm{~mm}$ on mount Pico, with $2350 \mathrm{~m}$ altitude the highest point of the archipelago. Santa Maria is situated south of São Miquel (Fig. 1) and is one of the smallest islands, measuring $96.89 \mathrm{~km}^{2}$. It has a population of c. 5.500 inhabitants, and a highest point of $590 \mathrm{~m}$ (source Wikipedia). The Azores are a major stronghold for remnants of the Tertiary evergreen forest, laurisilva, formerly widely distributed in southern Europe. These remnants are favourable habitats for foliicolous lichens (VAN DEN BOOM, 2016). So far relatively few lichens or lichenicolous fungi are recorded from Santa Maria (АРтROот et al., 2010; Gabriel, 2008).

At a first glance, the Azores seem to be well explored lichenologically. The first detailed list of li- chens was by Tavares (1941). Schumm (2008) and Schumm \& Aptroot (2013) present numerous macrolichens. Recent studies include Breuss (2018, 2019), ETAyo (2018), van DEN Boom (2016), van DEN Boom \& Alvarado (2019), van den Boom \& Magain (2020). However, the few published systematic studies, about the genus Usnea (CLERC, 2006), the genus Heterodermia (Moberg \& Purvis, 1997) and the genus Anisomeridium (VAN DEN BoOM, 2015), show that the taxonomical knowledge is still insufficient. In fact the crustose lichens as well as many macrolichen genera are in need of revision for this archipelago. In the summer of 2019, the author and his wife made a one-week excursion to Santa Maria, visiting 27 sites of lichenological interest, and collected lichens and lichenicolous fungi. Here we present a list of all 177 encountered species, which includes 18 species newly recorded for the Azores archipelago.

\section{MATERIALS AND METHODS}

All specimens have been studied by conventional macro- and microscopical techniques with hand-cut sections of the material mounted in tap water. Amyloid reactions were tested using Lugol's iodine solu- 
tion (K/I). Voucher specimens are kept in the herbarium of $\mathrm{P}$. van den Boom, a few specimens are deposited in the herbarium of Berlin Botanical Garden and Botanical Museum (B). Distribution data in Macaronesia were taken from the checklists of the Azores (Aptroot et al., 2010; GABRIEL, 2008) as well as more recently published papers, VAN DEN BOOM (2016), Etayo (2018), van den Boom \& Alvarado (2019), Guzow-Krzeminska et al. (2019). Species newly recorded from the Azores are marked with an asterisk. Fig. 1 gives a map of the Azores; Santa Maria is in the southeasternmost part. Fig. 2 presents habitus pictures of some of the more interesting species: Amandinea polyspora, Biatora britannica, Coenogonium tavaresianum, Endohyalina ericina, Orcularia insperata and Schismatomma graphidioides.

\section{COLLECTING SITES}

1. NE side of Vila Do Porto, Reserva Florestal de Valverde, mixed trees and shrubs, dominated by Thuja trees. $36^{\circ} 57.52^{\prime} \mathrm{N}, 25^{\circ} 08.06^{\prime} \mathrm{W}, 135 \mathrm{~m}$, 12 September 2019.

2. N of Vila Do Porto, Anjos, wall of acidic stones and two long rows of Metrosideros trees in field. $37^{\circ} 00.37^{\prime} \mathrm{N}, 25^{\circ} 09.23^{\prime} \mathrm{W}, 20 \mathrm{~m}, 12$ September 2019 .

\section{C \\ $\{\mathrm{Fi}$}
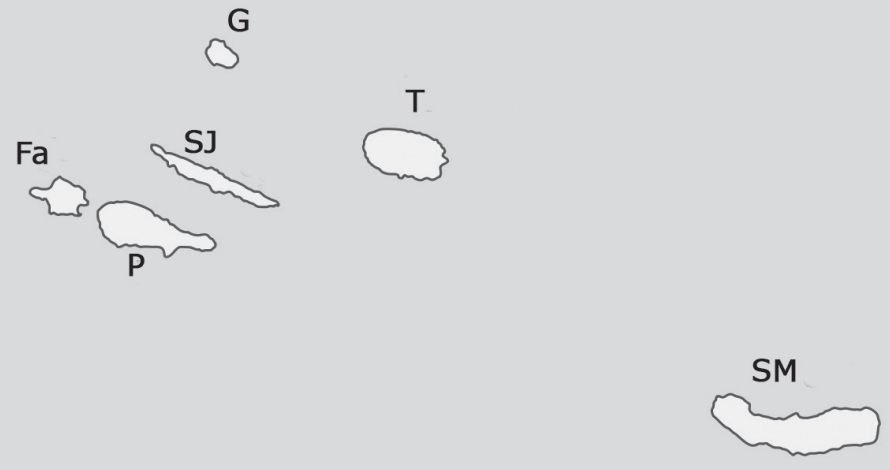

SMa

Fig. 1. The islands of the Azores archipelago. C - Corvo, Fi - Floris, Fa - Faial, G - = Graciosa, P - Pico, SJ - São Jorge, SM São Miguel, SMa - Santa Maria, T - Terceira 

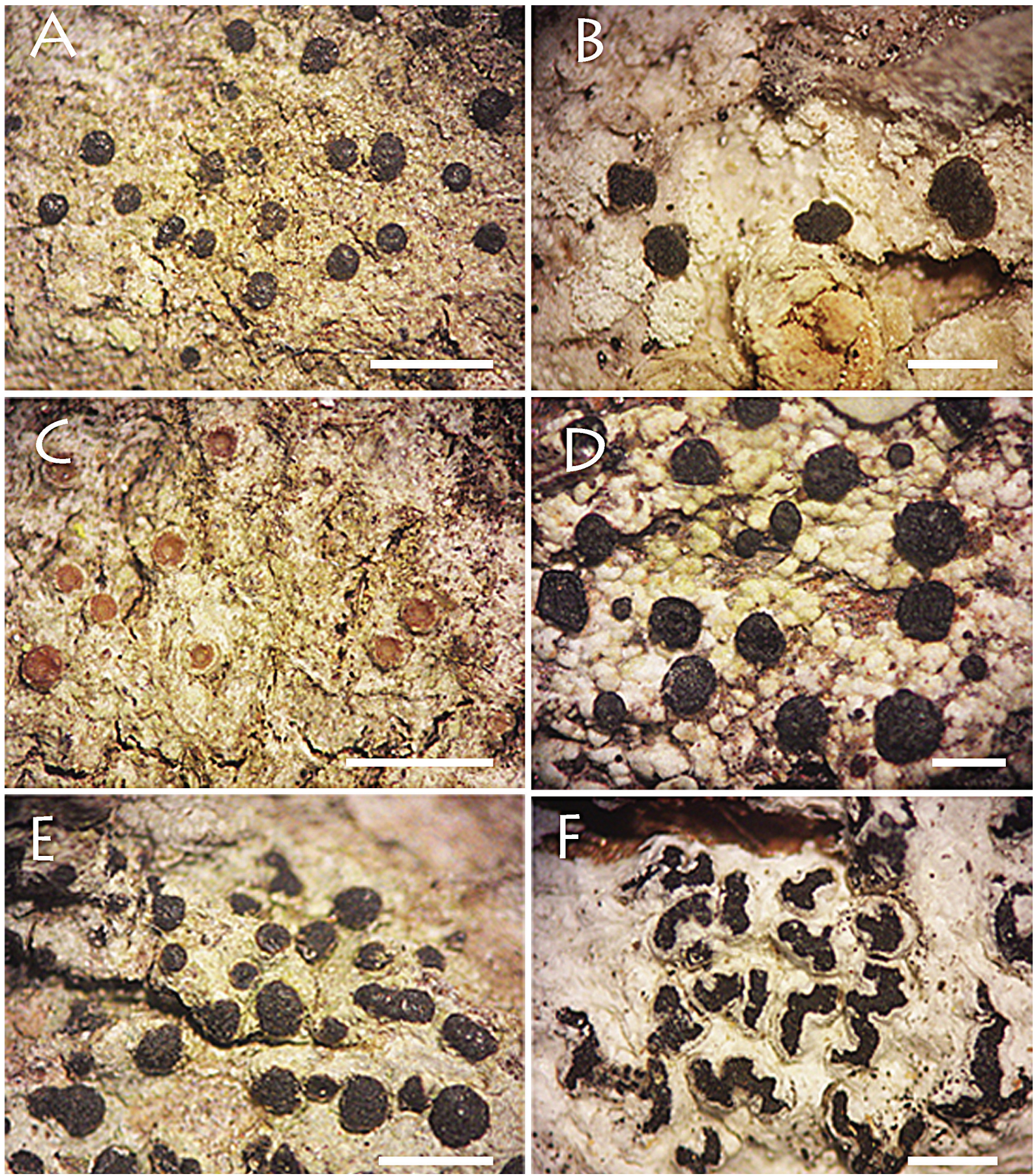

Fig. 2. Lichens from Santa Maria. A - Amandinea polyspora 58611, scale $=1 \mathrm{~mm}$; B - Biatora britannica 58779 , scale $=$ $1 \mathrm{~mm} ; \mathrm{C}-$ Coenogonium tavaresianum 58608, scale $=1 \mathrm{~mm} ; \mathrm{D}-$ Endohyalina ericina 59003, scale $=0.5 \mathrm{~mm} ; \mathrm{E}-$ Orcularia insperata 58976, scale $=1 \mathrm{~mm} ; \mathrm{F}-$ Schismatomma graphidioides 58617 , scale $=0.5 \mathrm{~mm}$

Fátima, old, neglected barn, on $\mathrm{N}$ exposed wall of acidic stones and on some Pittosporum trees along field. $37^{\circ} 00.11^{\prime} \mathrm{N}, 25^{\circ} 06.25^{\prime} \mathrm{W}, 250 \mathrm{~m}$, 13 September 2019.
10. NE of São Pedro, E of road ER-2-2, Chã do João Tolmé, trail to the east in forest, mature Cryptomeria and Pittosporum trees. $36^{\circ} 59.52^{\prime} \mathrm{N}, 25^{\circ}$ 06.16’ W, 230 m, 13 September 2019. 
11. S of São Pedro, along road ER-2-2, row of mature palm trees. $36^{\circ} 58.70^{\prime} \mathrm{N}, 25^{\circ} 07.42^{\prime} \mathrm{W}$, 160 m, 14 September 2019.

12. E of São Pedro, along secondary road from Ribeira do Engenho to Almagreira, area of Monteiro, wood of fence post and small group of trees in meadow. $36^{\circ} 58.77^{\prime} \mathrm{N}, 25^{\circ} 06.48^{\prime} \mathrm{W}, 250 \mathrm{~m}$, 14 September 2019.

13. NE of Almagreira, Pico Alto, along small road to the top (Pico Alto), c. $800 \mathrm{~m}$ from crossing with main road (ER-1-2), roadside Cryptomeria trees along forest. $36^{\circ} 58.67^{\prime} \mathrm{N}, 25^{\circ} 05.27^{\prime} \mathrm{W}, 460 \mathrm{~m}$, 14 September 2019.

14. NE of Almagreira, Pico Alto, along small road to the top (Pico Alto), c. $500 \mathrm{~m}$ from crossing with main road (ER-1-2), Erica at roadside and along field. $36^{\circ} 58.57^{\prime} \mathrm{N}, 25^{\circ} 05.18^{\prime} \mathrm{W}, 465 \mathrm{~m}$, 14 September 2019.

15. $\mathrm{N}$ of Vila do Porto, road to Anjos, c. $1 \mathrm{~km}$ SE of village (Anjos), acidic outcrops in field. $36^{\circ} 59.86^{\prime}$ N, 25 08.89’ W, 90 m, 14 September 2019.

16. $\mathrm{N}$ of Vila do Porto, road to Anjos, c. $500 \mathrm{~m} \mathrm{SE}$ of village, near picnic area, acidic outcrops in field, steep outcrops along road and a Metrosideros tree. $37^{\circ} 00.20^{\prime} \mathrm{N}, 25^{\circ} 09.10^{\prime} \mathrm{W}, 50 \mathrm{~m}, 14$ September 2019.

17. E of Vila do Porto, road ER-1-2, from Almagreira to Santo Espirito, Miradouro das Fontinhas, small picnic area (roadside), steep rock face and forest with Cryptomeria. 36 $57.95^{\circ}$ N, 25 04.73' W, 380 m, 15 September 2019.

18. SE of the island, along road ER-1, between Florestal das Fontinhas and Santo Espirito, just W before Santo Espirito, forest with Cryptomeria. $36^{\circ}$ 57.46' N, 250.97' W, 400 m, 15 September 2019.

19. S of Santo Espirito, S of Maia, Ponta do Castelo, near lighthouse, vertical and overhanging acidic outcrops. $36^{\circ} 55.79^{\prime} \mathrm{N}, 25^{\circ} 01.05^{\prime} \mathrm{W}, 100 \mathrm{~m}$, 15 September 2019.

20. SE of the island, along road ER-1, between Santo Espirito and Castelo, Calheta, near house and small forest, steep soil bank and Buxus in garden. $36^{\circ} 56.72^{\prime}$ N, $25^{\circ} 01.96^{\prime}$ W, 255 m, 15 September 2019 .

21. E of Almagreira, along road ER-1-2, to Santo Espirito, Reserva Florestal das Fontinhas, very big picnic area, few Quercus trees, many Camellia shrubs and a forest with mature Cryptome- ria trees. $36^{\circ} 57.61^{\prime} \mathrm{N}, 25^{\circ} 04.40^{\prime} \mathrm{W}, 355 \mathrm{~m}$, 16 September 2019.

22. Santa Barbara, centre, solitary Camellia shrub near brooklet. $36^{\circ} 59.07^{\prime} \mathrm{N}, 25^{\circ} 04.12^{\prime} \mathrm{W}$, 170 m, 16 September 2019.

23. NNE of Santa Barbara, near Pico do Norte, N of picnic area, just $\mathrm{S}$ of Norte, small forest with mainly Myrica faya, along secondary road. $37^{\circ} 00.18^{\prime} \mathrm{N}$, $25^{\circ} 03.77^{\prime} \mathrm{W}, 225 \mathrm{~m}, 16$ September 2019.

24. SE of Santa Barbara, S side of the main road to São Lourenço, Pico Vermelho, Poço da Pedreira, Erica forest with view point. $36^{\circ} 58.89^{\prime} \mathrm{N}, 25^{\circ}$ 03.67' W, 305 m, 16 September 2019.

25. S of Malbusca, Piedade, trail to Ponta da Malbusca, field with shrubs, mainly Erica and volcanic outcrops. $36^{\circ} 56.17^{\prime} \mathrm{N}, 25^{\circ} 04.35^{\prime} \mathrm{W}$, $160 \mathrm{~m}, 16$ September 2019.

26. S of Santo Espirito, S of Cardal, trail in field with a row of small trees, Erica and Myrica faya. $36^{\circ}$ $56.15^{\prime} \mathrm{N}, 25^{\circ} 02.85^{\prime} \mathrm{W}, 240 \mathrm{~m}, 17$ September 2019.

27. S of Santo Espirito, S of Cardal, trail in forest along brooklet, with mainly Picconia and Pittosporum trees. $36^{\circ} 56.09^{\prime} \mathrm{N}, 25^{\circ} 03.01^{\prime} \mathrm{W}$, 180 m, 17 September 2019.

\section{ANNOTATED SPECIES LIST}

The numbers in brackets correspond to the collecting sites listed above; they are followed by the substrate and the collection number of the voucher.

Abrothallus hypotrachynae Etayo \& Diederich - (26) on Hypotrachyna on Erica, 59055; (26) on Hypotrachyna on Myrica, 59066; (26) on Hypotrachyna on Erica, 59056.

Acarospora umbilicata Bagl. - (25) on volcanic rock, 59043.

Acrocordia macrospora A. Massal. - (16) on volcanic rock, 58855.

Alyxoria mougeotii (A. Massal.) Ertz, Frisch \& G. Thor-(16) on volcanic rock, 58849.

Amandinea pelidna (Ach.) Fryday \& L. Arcadia (15) on volcanic rock, 58838.

Amandinea polyspora (Willey) E. Lay \& P. May (1) on Thuja, 58611; (3) on stump, 58681 [Fig. 2A].

Amandinea punctata (Hoffm.) Coppins \& Scheid. (2) on stone on soil, 58670. 
Anisomeridium robustum Orange, Coppins \& Aptroot - (4) on Metrosideros, 58718.

*Arthonia anglica Coppins - (1) on Pittosporum, 58647.

Arthonia atra (Pers.) A. Schneid. - (2) on Metrosideros, 58679; (22) on Camellia, 58971; (25) on Erica, 59023.

Arthonia elegans (Ach.) Almq. - (1) on tree, 58636; (27) on Myrica, 59083.

Arthonia stellaris Kremp. - (1) on Grevillea, 58628;

(3) on Pittosporum, 58701; (22) on Camellia, 58969; (23) on Myrica, 58975; (25) on Erica, 59012; (26) on Myrica, 59068.

Aspicilia cf. tenuis (H. Magn.) Owe-Larss. \& A. Nordin - (16) on volcanic rock, 58860.

Bacidia arceutina (Ach.) Arnold - (1) on Eucalyptus, 58615; (7) on wood of fence, 58704; (21) on wood of fence, 58967; (23) on tree, 58987.

Bacidia heterochroa (Müll. Arg.) Zahlbr. - (1) on Thuja, 58618, 58621; (1) on Hibiscus, 58632; (4) on Metrosideros, 58722; (8) on Rubus, 58763; (16) on Metrosideros, 58851; (20) on leaf of Buxus, 58917; (22) on Camellia, 58970; (23) on Myrica, 58978; (25) on Erica, 59011; (26) on Myrica, 59049, 59071; (27) on Eucalyptus, 59011; (27) on Myrica, 59081.

Bacidina apiahica (Müll. Arg.) Vězda - (13) on needles of Cryptomeria, 58816; (17) on needles of Cryptomeria, 58874; (20) on leaves of Buxus, 58923; (21) on needles of Cryptomeria, 58958.

Bacidina chloroticula (Nyl.) A.L. Sm. - (3) on Erica, 58708; (20) on leaves of Buxus, 58928.

Bacidina pallidocarpa van den Boom \& Magain (21) on wood of fence, 58966.

Baeomyces rufus (Huds.) Rebent. - (21) terricolous, 58962.

*Biatora britannica Printzen, Lumbsch \& Orange (1) on Pittosporum, 58779 [Fig. 2B].

Biatoropsis usnearum Räsänen s.1. - (9) on Usnea on Pittosporum, 58980.

Blastenia crenularia (With.) Arup, Søchting \& Frödén - (2) on stone on soil, 58668; (15) on volcanic rock, 58837; (16) on volcanic rock, 58853, 58859.

*Botryolepraria lesdainii (Hue) Canals et al. - (21) on stone of wall, 58961.

Buellia mediterranea Giralt - (12) on wood of fence, 58809.
*Buellia schaereri De Not. - (3) on stump, 58684.

*Buellia stellulata (Taylor) Mudd - (2) on stone on soil, 58665; (15) on volcanic rock, 58840; (16) on volcanic rock, 58856 .

Byssoloma croceum Sérus. \& Puntillo - (3) on Erica, 58709; (21) on leaf of Camellia, 59107; (24) on Erica, 58993; (25) on Erica, 59013, 59030.

Byssoloma fuscum van den Boom - (6) on needles of Cryptomeria, 58736; (21) on leaves of Buxus, 58944; (24) on Erica, 59007.

Byssoloma kakouettae (Sérus.) Lücking \& Sérus. (10) on leaf, 58778; (17) on needles of Cryptomeria, 58873; (18) on needles of Cryptomeria, 58879, 58882; (21) on leaf of Camellia, 58938, $58939,58940,58947,58948$; (21) on needles of Cryptomeria, 58960.

Byssoloma leucoblepharum (Nyl.) Vain. em. R. Sant. - (27) on leaf of Picconia, 59090.

Byssoloma maderense Breuss - (3) on Erica, 58698.

Byssoloma marginatum (Arnold) Sérus. - (21) on needles of Cryptomeria, 58959; (21) on leaves of Buxus, 58943.

Byssoloma subdiscordans (Nyl.) P. James - (6) on Pittosporum, 58738, 58741; (6) on needles of Cryptomeria; (21) on leaves of palm; (21) on leaf of Camellia, 58937, 58946.

Candelariella vitellina (Hoffm.) Müll. Arg. - (2) on stone on soil, 58671; (16) on volcanic rock, 58870; (25) on volcanic rock, 59042.

Canoparmelia amabilis Heiman \& Elix - (12) on tree, 58801.

Canoparmelia caroliniana (Nyl.) Elix \& Hale - (5) on wood of bench, 58726 .

Cladonia coniocraea (Flörke) Spreng. - (6) on Pittosporum, 58746; (21) on Cryptomeria, 58955.

Cladonia nana Vain. - (3) terricolous, 58712.

Cladonia ochrochlora Flörke - (13) on Cryptomeria, 58813.

Cladonia peziziformis (With.) J.R. Laundon - (3) terricolous, 58717.

Cladonia ramulosa (With.) J.R. Laundon - (3) on stump, 58683.

Cladonia stereoclada Abbayes - (3) terricolous, 58715; (17) on volcanic rock, 58878.

Clandestinotrema antoninii (Purvis \& P. James) Rivas Plata, Lücking \& Lumbsch - (7) on Erica, 58757, 58758; (14) on Erica;

Cliomegalaria symmictoides van den Boom \& Al- 
varado - (3) on Pinus, 58695; (3) on Pittosporum; (12) on tree, 58800; (24) on Erica, 58996, 58999; (27) on Eucalyptus, 59102.

Cliostomum griffithii (Sm.) Coppins - (24) on Erica, 59010; (25) on Erica, 59016.

Coenogonium tavaresianum (Vězda) Lücking, Aptroot \& Sipman - (1) on Thuja, 58608 [Fig. 2C].

Coscinocladium gaditanum (Clemente) A. Crespo, Llimona \& D. Hawksw. - (9) on stone of wall, 58769.

*Didymocyrtis cladoniicola (Diederich, Kocourk. \& Etayo) Ertz \& Diederich - (1) on Ramalina on Thuja, 58646.

Diploicia canescens (Dicks.) A. Massal. - (9) on stone of wall, 58770; (16) on volcanic rock, 58862.

Diploicia canescens (Dicks.) A. Massal. ssp. australasica Elix \& Lumbsch - (19) on volcanic rock, 58904.

Diploschistes actinostomus (Ach.) Zahlbr. - (25) on volcanic rock, 59035.

Dirina insulana (Tav.) Tehler - (19) on volcanic rock, 58913.

Dirinaria applanata (Fée) D.D. Awasthi - (12) on wood of fence post, 58805; (26) on Erica, 59047.

Enchylium tenax (Sw.) Gray - (19) on mortar, 58892.

Endococcus propinquus (Körb.) D. Hawksw. - (15) on Buellia on volcanic rock, 58839.

Endohyalina ericina (Nyl.) Giralt, van den Boom \& Elix - (23) on Myrica, 58974; (24) on Erica, 58995, 59003; (25) on Erica, 59028 [Fig. 2D].

Enterographa crassa (DC.) Fée - (9) on stone of wall, 58767.

Fellhanera azorica van den Boom - (18) on needles of Cryptomeria; (20) on leaves of Buxus; (27) on leaves of Picconia.

Fellhanera seroexspectata Sérus. - (21) on leaf of Camellia, 58935.

*Fellhaneropsis myrtillicola (Erichsen) Sérus. \& Coppins - (13) on needles of Cryptomeria, 58817, 58819.

Flavoparmelia caperata (L.) Hale - (3) on stump, 58687.

Flavoplaca citrina (Hoffm.) Arup, Frödén \& Søchting - (3) on stump; (19) on calcareous stone, on soil, 58898.

Flavoplaca flavocitrina (Nyl.) Arup, Frödén \&
Søchting - (26) on volcanic rock, 59045.

Flavoplaca marina (Wedd.) Arup, Frödén \& Søchting - (2) on stone on soil, 58664.

Fulvophyton sorediata (Sparrius, L., P. James \& M.A. Allen) Tehler \& van den Boom - (19) on volcanic rock, 58907.

Graphis scripta (L.) Ach. - (1) on tree, 58634.

Gyalecta schisticola Werner-(16) on volcanic rock, 58854.

*Gyalectidium membranaceum Sérus. - (6) on leaf of Pittosporum, 58742; (17) on needles of Cryptomeria, 58875; (21) on leaf of Camellia, 58939.

Gyalolechia flavorubescens (Huds.) Søchting, Frödén \& Arup - (1) on Thuja, 58643; (1) on tree, 58659; (4) on Metrosideros, 58721, 58725.

Herteliana gagei (Sm.) J.R. Laundon - (7) on volcanic rock, 58759.

Heterodermia albicans (Pers.) Swinscow \& Krog (15) on volcanic rock, 58841.

Heterodermia leucomela (Fée) Swinscow \& Krog (4) on stone of wall, 58724; (11) on palm, 58794.

Heterodermia obscurata (Nyl.) Trevisan - (1) on Pittosporum, 58649.

Hyperphyscia adglutinata (Flörke) Mayrh. \& Poelt (20) on leaves of Buxus, 58926.

Hypotrachyna rockii (Zahlbr.) Hale - (3) on Erica, 58694; (24) on Erica, 59001; (26) on Myrica, 59061.

Lecania cyrtella (Ach.) Th.Fr. - Loc. (1) on Hibiscus, 58630; (18) on needles of Cryptomeria, 58883; (21) on needles of Cryptomeria, 58957.

Lecania naegelii (Hepp) Diederich \& van den Boom - (22) on Camellia, 58972.

*Lecania nigra van den Boom \& Ertz - (19) on volcanic rock, 58909.

Lecanora albella (Pers.) Ach. - (1) on Thuja, 58619; (3) on stump, 58682; (12) on wood, 58812; (23) on Myrica, 58979; (25) on Erica, 59029.

Lecanora chlarotera Nyl. - (23) on Myrica, 58973.

Lecanora gangaleoides Nyl. -(19) on volcanic rock, 58901.

Lecanora hybocarpa (Tuck.) Brodo - (1) on shrub 58655; (1) on Thuja, 2716; (8) on Rubus, 58764; (26) on Myrica, 59072.

Lecanora strobilina (Spreng.) Kieff. - (25) on Erica, 59025. 
Lecanora subrugosa Nyl. - (2) on Metrosideros, 58673.

*Lecanora subsaligna M. Brand \& van den Boom (3) on stump, 58685; (26) on Erica, 59059.

Lecidella asema (Nyl.) Knoph \& Hertel - (2) on stone on soil, 58672; (19) on volcanic rock, 58900.

Lepraria finkii (B. de Lesd.) R.C. Harris - (17) on Platanus, 58877; (27) on Picconia, 59093.

Lepraria incana (L.) Ach. - (6) on needles of Cryptomeria, 58740; (10) on Cryptomeria, 58782.

Leprocaulon microscopicum (Vill.) Gams ex D. Hawksw. - (19) on volcanic rock, 58895.

Leptogium brebissonii Mont. - (3) on Pittosporum, 58691; (9) on Pittosporum, 58774; (12) on tree, 58802.

Leptogium cyanescens (Rabenh.) Körb. - (1) on Eucalyptus, 58651.

*Lichenoconium lichenicola (P. Karst.) Petr. et Syd. - (12) on Buellia on wood of fence, 58835.

Lichenoconium usneae (Anzi) D. Hawksw. - (19) on Heterodermia leucomela on volcanic rock, 58910; (27) on Heterodermia leucomela on Myrica, 59085.

Lichenodiplis lecanorae (Vouaux) Dyko \& D. Hawksw. - (12) on Caloplaca s.l. on wood of fence, 58811; (25) on Caloplaca s.1. on Erica, 59017.

*Lichenodiplis pertusariicola (Nyl.) Diederich - (27) on Pertusaria on Eucalyptus, 59104.

Lobaria immixta Vain. - (6) on Pittosporum, 58743.

Lobaria virens (With.) J.R. Laundon - (6) on Pittosporum, 58745.

*Marchantiana asserigena (J. Lahm) Søchting \& Arup - (24) on Erica, 58994; (25) on Erica, 59015; (26) on Erica, 59053.

Massalongia carnosa (Dicks.) Körb. - (21) terricolous, 58963.

Megalaria albocincta (Degel.) Tønsberg - (14) on Erica, 58824, 58828.

Megalaria pulverea (Borrer) Hafellner \& E. Schreiner - (7) on Erica, 58756.

Minutoexcipula marina V. Atienza - (1) on Pertusaria on Grevillea, 58625; (27) on Pertusaria on Myrica, 59087.

Moelleropsis nebulosa (Hoffm.) Gyeln. - (20) terricolous, 58914.

Muellerella lichenicola (Sommerf.) D. Hawksw. (3) on Lecanora on stump, 58686; (12) on Le- canora on wood of fence, 59024.

Normandina pulchella (Borrer) Nyl. - (3) on Pittosporum, 58692; (6) on needles of Cryptomeria, 58737; (13) on needles of Cryptomeria, 58821.

Ochrolechia parella (L.) A. Massal. - (2) on stone on soil, 58663.

*Opegrapha foreaui (Moreau) Hafellner \& R. Sant. (27) on Heterodermia on Myrica, 59084.

*Opegrapha melanospila Müll. Arg. - (9) on Parmotrema on Pittosporum 58772.

Orcularia insperata (Nyl.) Kalb \& Giralt - (2) on Metrosideros, 58677; (16) on Metrosideros, 58976 (Fig. 2E).

Parmelinopsis minarum (Vain.) Elix \& Hale - (3) on Erica, 58699; (27) on Psidium, 59095.

Parmotrema perlatum (Huds.) M. Choisy - (1) on Thuja, 58612; (1) on tree, 58660; (7) on Erica, 58754; (20) on leaves of Buxus, 58903.

Parmotrema reticulatum (Taylor) M. Choisy - (3) on Erica; (27) on Psidium, 59096.

Parmotrema robustum (Degel.) Hale - (14) on Erica, 58832.

Parmotrema subisidiosum (Müll. Arg.) Hale - (12) on tree, 58803; (26) on Erica, 59057; (26) on volcanic rock, 59076.

Pectenia atlantica (Degel.) P.M. Jørg., L. Lindblom, Wedin \& S. Ekman - (6) on Pittosporum, 58744.

Pectenia ligulata (P. James) P.M. Jørg., L. Lindblom, Wedin \& S. Ekman - (19) on volcanic rock, 58912; (26) on Myrica, 59079.

Peltigera hymenina (Ach.) Delise ex Duby - (7) terricolous, 58751; (21) terricolous, 58964.

*Pertusaria coronata (Ach.) Th. Fr. - (19) on volcanic rock, 58894.

Pertusaria pseudocorallina (Lilj.) Arnold - (15) on volcanic rock, 58844.

Pertusaria pustulata (Ach.) Duby - (26) on Myrica, 59063.

Phaeographis dendritica (Ach.) Müll. Arg. - (23) on tree, 58981; (27) on Eucalyptus, 59089, 59099.

Phaeophyscia hirsuta (Mereschk.) Essl. - (25) on volcanic rock, 59036.

Phaeophyscia orbicularis (Neck.) Moberg - (11) on palm, 58792; (16) on volcanic rock, 58863.

Physcia adscendens H. Olivier - (1) on shrub, 58656; (2) on Metrosideros, 58675; (27) on Myrica, 59082. 
Physcia atrostriata Moberg - (1) on tree, 58657.

Physcia erumpens Moberg - (1) on tree, 58653, 58654; (11) on palm, 58788.

Physcia tribacioides Nyl. - (20) on leaves of Buxus, 58925; (26) on volcanic rock, 59046.

Porina aenea (Wallr.) Zahlbr. - (20) on leaves of Buxus, 58934.

Porina coralloidea P. James - (24) on Erica, 59004; (25) on Erica, 59034.

Porina leptalea (Durieu \& Mont.) A.L. Sm. - (21) on tree-fern, 58942.

Porina leptospora (Nyl.) A.L. Sm. - (1) on Thuja, 58610; (19) on volcanic rock (cf.), 58911; (20) on leaves of Buxus, 58930; (23) on Myrica, 58983; (24) on Erica, 58990; (25) on Erica, 59020, 59031, 59040.

Porina tetramera (Malme) R. Sant. - (6) on needles of Cryptomeria, 58734; (13) on needles of Cryptomeria, 58815; (17) on needles of Cryptomeria, 58876; (21) on leaf of Camellia, 58936.

Porpidia crustulata (Ach.) Hertel \& Knoph - (7) on wood of fence post, 58747.

*Pronectria angulospora Etayo - (26) on Heterodermia on Myrica, 59077.

Pseudocyphellaria aurata (Ach.) Vain. - (3) on Pittosporum, 58690; (6) on needles of Cryptomeria, 58735; (27) on Myrica, 59088.

Pyrenula dermatodes (Borrer) Schaer. - (14) on Erica, 58836, 59106.

Pyrenula laevigata (Pers.) Arnold - (1) on Grevillea, 58624.

Pyrenula macrospora (Degel.) Coppins \& P. James (1) on tree, 58637; (9) on Pittosporum, 58773; (12) on tree, 58798.

Pyrrhospora quernea (Dicks.) Körber - (1) on Eucalyptus, 58614; (3) on stump, 58688.

Pyxine sorediata (Ach.) Mont. - (16) on volcanic rock, 58867.

Pyxine subcinerea Stirt. - (1) on tree, 58640; (1) on shrub, 58658; (2) on Metrosideros, 58678; (19) on volcanic rock, 58899; (26) on volcanic rock, 59044; (26) on Erica, 59054, 59058.

Ramalina azorica Schumm \& Aptroot - (19) on volcanic rock, 58887.

Ramalina chondrina J. Steiner - (9) on Pittosporum, 58777; (26) on Myrica, 59052.

Ramalina lacera (With.) J.R. Laundon - (2) on Metrosideros, 58674; (4) on stone of wall, 58719.
Ramalina lusitanica H. Magn. - (16) on Metrosideros, 58850.

Ramalina peruviana Ach. - (1) on Hibiscus, 58631; (1) on tree, 58662; (9) on Pittosporum, 58775; (12) on wood of fence (cf.), 58808.

Ramalina pusilla Le Prév. ex Duby - (1) on tree, 58661; (8) on Pittosporum, 58762; (12) on wood of fence post, 58806.

Ramalina requienii (De Not.) Jatta - (2) on stone on soil, 58669; (9) on stone of wall, 58768; (11) on palm, 58793; (16) on volcanic rock, 58846, 58858; (19) on volcanic rock, 58891.

Ramalina siliquosa (Huds.) A.L. Sm. - (19) on volcanic rock, 58885, 58890.

Ramalina subpusilla (Nyl.) Krog \& Swinscow - (3) on Pittosporum, 58689; (8) on Pittosporum, 58765; (23) on Myrica, 58982; (23) on tree, 58988; (26) on Myrica, 59051; (27) on Eucalyptus, 59103.

Rinodina anomala (Zahlbr.) H. Mayrhofer \& Giralt - (1) on Thuja, 58623, 58645; (25) on Erica, 59022, 59032.

Rinodina beccariana Bagl. v. beccariana - (1) on stone on soil, 58667; (16) on volcanic rock, 58864, 58869,$58871 ;$ (25) on volcanic rock, 59039.

*Rinodina boleana Giralt \& H. Mayrhofer - (25) on Erica, 59021.

Rinodina colobinoides (Nyl.) Zahlbr. - (1) on tree, 58641.

Roccella allorgei Abbayes - (19) on volcanic rock, 58888.

Roccella elisabethae Tehler - (16) on volcanic rock, 58857.

Roccella fuciformis (L.) DC. - (11) on palm, 58787; (19) on volcanic rock, 58902.

Roccella tinctoria DC. - (11) on palm, 58795; (16) on volcanic rock, 58847.

Roccella tuberculata Vain. - (19) on volcanic rock, 58905.

Sagiolechia atlantica Henssen - (16) on volcanic rock, 58848; (19) on volcanic rock, 58897.

Schismatomma graphidioides (Leight.) Zahlbr. - (1) on Thuja, 58617 [Fig. 2F].

Scoliciosporum umbrinum (Ach.) Arnold - (3) on stump, 58713, 58714; (3) on Pittosporum, 58702; (3) on Erica, 58711; (7) on wood of fence, 58748, 58749; (12) on wood of fence, 58810; (25) on Erica, 59014. 
Scytinium teretiusculum (Wallr.) Otálora, P.M. Jørg. \& Wedin - (1) on Eucalyptus, 58652.

Solitaria chrysophthalma (Degel.) Arup, Søchting \& Frödén - (2) on Metrosideros, 58676.

Sphinctrina tubiformis A. Massal. - (1) on Pertusaria on tree, 58638; (26) on Pertusaria on Myrica, 59075.

Sticta canariensis (Ach.) Bory - (7) on Vaccinium, 58752.

Sticta limbata (Sm.) Ach. - (7) on Vaccinium, 58753.

Stigmidium triebeliae Etayo - (2) on Parmotrema reticulatum on stone on soil, 58666; (15) on Parmotrema reticulatum on volcanic rock, 58842.

Syncesia myrticola (Fée) Tehler - (1) on Thuja, 58616; (8) on Pittosporum, 58761; (11) on palm, 58790; (12) on tree, 58797; (27) on Psidium, 59094.

Teloschistes chrysophtalmus (L.) Th.Fr. - (1) on Camellia, 58642.

Teloschistes flavicans (Sw.) Norman - (4) on stone of wall, 58720.

Tephromela atra (Huds.) Hafellner - (16) on volcanic rock, 58845, 58866; (25) on volcanic rock, 59038.

Thelocarpon microsporum van den Boom - (6) on Cryptomeria, 58730; (21) on Cryptomeria, 58952.

Thelotrema lepadinum (Ach.) Ach. - (10) on Pittosporum, 58780, 58783; (14) on Erica, 58827; (21) on tree-fern, 58941; (24) on Erica, 59002; (26) on Myrica, 59073.

Toniniopsis aromatica (Sm.) Kistenich, Timdal, Bendiksby \& S. Ekman - (16) on Aspicilia s.l. on volcanic rock, 59110; (19) on volcanic rock, 58908, 58909a.

Trapelia corticola Coppins \& P. James - (6) on Cryptomeria, 58733.

Tremella ramalinae Diederich - (9) on Ramalina on Pittosporum, 58776.

\section{DISCUSSION}

The checklist of Aptroot et al. (2010) includes 56 lichen species for Santa Maria, mainly macro lichens from genera such as Heterodermia, Lobaria, Parmotrema, Ramalina and Roccella, usually common species occurring on nearly all of the other islands. Only a very few rare species in the checklist are known from Santa Maria and not from other islands in the archipelago: Cetrelia olivetorum (Nyl.) W.L. Culb. $\&$ C.F. Culb. and Lecanora leprosa Fée. No lichenicolous fungi for Santa Maria can be found in this checklist.

A recent survey on the genus Cladonia in the Azores by Pino-Bodas et al. (2017) treated 29 species in detail. An annotated list of all previously reported Cladonia species from the Azores is included and 24 further species are mentioned of which they regard some as doubtful. The only recorded species from Santa Maria in that study is Cladonia caespiticia (Pers.) Flörke. In Aptroot et al. (2010) are recorded 3 Cladonia species for the island and in this study 6 species.

For this study, in total 500 specimens of lichens and lichenicolous fungi have been collected, on all available substrata, in 27 localities all over the island. At least 177 species could be identified (annotated species list). Of these, 18 records are new to the Azores archipelago. Twenty-one foliicolous species are recorded on leaves or on needles. Not any foliicolous record for Santa Maria can be find in the recent study about foliicolous lichens in Macaronesia (van den Boom 2021). One species from that recent study, newly described, Fellhanera azorica van den Boom, has been found and is listed here.

Several specimens still remain unidentified, for instance because there are no identification keys or reliable treatments (e.g., in the case of Bacidia, Bacidina, Buellia, Fissurina, Graphis, Lecanora, Lepraria, Opegrapha and lichenicolous fungi). This indicates that the lichen biota on Santa Maria is certainly more diverse than reported here and additional records can be expected, including newly described species.

\section{ACKNOWLEDGEMENT}

I would like to thank Dr. Harrie Sipman for doing a TLC analysis of some specimens, especially from Cladonia and Ramalina and for helpful comments on the manuscript. Two anonymous referees provided comments which improved the manuscript. Dr. Tor Tønsberg kindly did the TLC for Biatora britannica. Bern van den Boom discovered many of the lichenicolous fungi. 


\section{REFERENCES}

Aptroot A., Rodrigues A.F., Schumm F. CÂmara S., GABRIEL R., 2010: Lista dos líquenes e fungos liquenícolas (Fungi). List of lichens and lichenicolous fungi (Fungi). - In: Botges P.A.V., Costa A., Cunha R., Gabriel R., Gonçalves V., Martins A.F., Melo I., Parente M., Raposeiro P., Rodrigues P., Santos R.S., Silva L., Vieira P., Vieira V. (eds): Listagem dos Organismos Terrestres e Marinhos dos Açores. A list of the Terrestrial and Marine Biota from the Azores: 59-79. - Cascais.

BREuss O., 2018: New and remarkable lichen records from the Azores (São Miguel Island). - Herzogia, 31: 430-435.

Breuss O., [2018] 2019: Neue und bemerkenswerte Flechtenfunde von den Azoren (Insel São Miguel) II. - Österreichische Zeitschrift für Pilzkunde, 27: 31-36.

Clerc P., 2006: Synopsis of Usnea (lichenized Ascomycetes) from the Azores with additional information on species in Macaronesia. - Lichenologist, 38: 191-212.

EтAyo J., 2018: Lichenicolous fungi of the Azores islands. I. Terceira. - Ascomycete.org., 10: 107116.

Gabriel R., 2008: Base de dados da biodiversidade dos Açores. Líquenes. - Universidade dos Açores. http://www.azoresbioportal.angra.uac.pt/listagens.php?sstr=1\&lang=pt (visited 11.10.2020).

Guzow-Krzemińska B., Sérusiaux E., van deN Boom P.P.G., Brand A.M., Launis A., ŁubeK A., KuKWA M., 2019: Understanding the evolution of phenotypical characters in the Micarea prasina group (Pilocarpaceae) and descriptions of six new species within the group. - MycoKeys, 57: 1-30.
Moberg R., Purvis W., 1997: Studies on the lichens of the Azores. Part 4. The genus Heterodermia. - Symbolae Botanicae Upsalienses, 32(1): 187-194.

Pino-Bodas R., Ahti T., Stenroos S., 2017: Cladoniaceae of the Azores. - Herzogia, 30: 445-462.

Schumm F., 2008: Flechten Madeiras, der Kanaren und Azoren. - Wangen.

Schumm F., Aptroot A., 2013: Flechten Madeiras, der Kanaren und Azoren - Band 2 Ergänzungsband. - Wangen.

Tavares C.N., 1941: Algumas especies de Liquenes novas para os Açores. - Boletim da Sociedade Portuguesa de Ciências Naturais, 13, Suppl. 2: 232-234.

VAN DEN Boom P.P.G., 2015: Notes on the genus Anisomeridium (lichenized Ascomycotina) from Madeira and the Azores (Macaronesia). - Phytotaxa, 205(1): 65-70.

van DEN Boom P.P.G., 2016: Lichens and lichenicolous fungi of the Azores (Portugal), collected on São Miguel and Terceira with the descriptions of seven new species. - Acta Botanica Hungarica, 58(1-2): 199-222.

VAN DEN BOOM P.P.G., 2021: Foliicolous lichens and their lichenicolous fungi in Macaronesia and Atlantic Europe. - Bibliotheca Lichenologica 111: $1-197$.

van den Boom P.P.G., Alvarado P., 2019: Lichens and lichenicolous fungi of Faial (Azores, Portugal) with descriptions of three new species. Herzogia, 32: 421-437.

van den Boom P.P.G., Magain N., 2020: Three new lichen species from Macaronesia belonging in Ramalinaceae, with the description of a new genus. Plant and Fungal Systematics, 65(1): 167-175.

\section{DUOMENYS APIE KERPES IR LICHENOFILINIUS GRYBUS IŠ SANTA MARIJA SALOS (AZORU ARCHIPELAGAS)}

\section{Pieter P.G. VAN DEN BOOM}

\section{Santrauka}

Straipsnyje pateikiami duomenys apie šimtą septyniasdešimt septynias kerpių ir lichenofilinių grybų rūšis, aptiktas Santa Marija saloje (Azorų archipelagas, Portu- galija). Iš jų aštuoniolika rūšių yra naujos archipelagui. Sudarytas rūšių sąrašas su radavietemis ir jų substratais. Šešių retų rūšių pateiktos orginalios nuotraukos. 